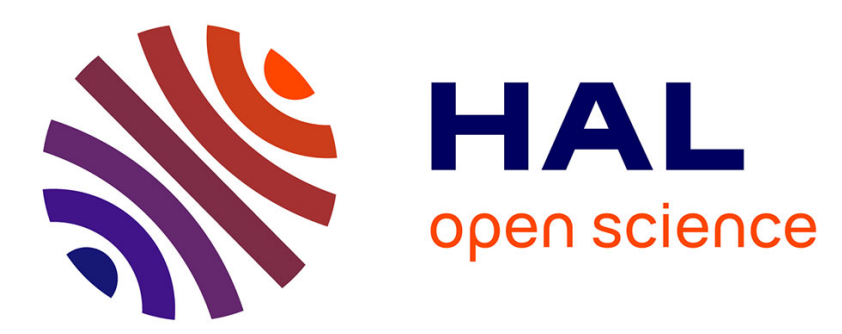

\title{
Robust pole placement controller design in LMI region for uncertain and disturbed switched systems
}

Badr Mansouri, Noureddine Manamanni, Kevin Guelton, Mohamed Djemai

\section{To cite this version:}

Badr Mansouri, Noureddine Manamanni, Kevin Guelton, Mohamed Djemai. Robust pole placement controller design in LMI region for uncertain and disturbed switched systems. Nonlinear Analysis: Hybrid Systems, 2008, 2 (4), pp.1136-1143. 10.1016/j.nahs.2008.09.010 hal-00793234

\section{HAL Id: hal-00793234 \\ https://hal.science/hal-00793234}

Submitted on 21 Feb 2013

HAL is a multi-disciplinary open access archive for the deposit and dissemination of scientific research documents, whether they are published or not. The documents may come from teaching and research institutions in France or abroad, or from public or private research centers.
L'archive ouverte pluridisciplinaire HAL, est destinée au dépôt et à la diffusion de documents scientifiques de niveau recherche, publiés ou non, émanant des établissements d'enseignement et de recherche français ou étrangers, des laboratoires publics ou privés. 


\title{
Robust pole placement controller design in LMI
}

\section{region for uncertain and disturbed switched}

\author{
systems
}

B. Mansouri*, N. Manamanni*, K. Guelton*, M. Djemai**

*CReSTIC, EA3804, University of Reims Champagne Ardenne

Moulin de la House BP1039, 51687 Reims Cedex 2, France

Tel : (+33) 326918386 fax : (+33) 326913106

\{badr.mansouri, noureddine.manamanni, kevin.guelton\}@univ-reims.fr

**ECS, ENSEA, 6 avenue du Ponceau, 95014 Cergy, France

djemai@ensea.fr

Corresponding author: N. Manamanni, noureddine.manamanni@ univ-reims.fr

\begin{abstract}
:
This paper concerns the state feedback control for continuous time, disturbed and uncertain linear switched systems with arbitrary switching rules. The main result of this work consists in getting an LMI (Linear matrix inequalities) condition guaranteeing a robust poles placement according to some desired specifications. Then, external disturbances attenuation with a fixed rate according to $\mathrm{H} \infty$ criterion is ensured. This is obtained thanks to the existence of a common quadratic Lyapunov function for all subsystems. Finally, an academic example illustrates the efficiency of the developed approach.
\end{abstract}

\section{Keywords:}

Hybrid dynamical systems, linear switched systems, switched control, quadratic Lyapunov function, LMI (Linear matrix inequalities), Hœ control. 


\section{Introduction}

Hybrid dynamic systems are defined as a set of continuous and discrete sub-systems interacting together. Thus, they associate continuous and discrete dynamic, as well as continuous and discrete control [2], [14], [15]. The hybrid dynamic systems (HDS) have many varied applications, one finds them in the control of mechanical systems, motorized industry, aeronautics, power electric converters, robotics...

The main difficulty in the definition of HDS is that the hybrid term is not restrictive; the interpretation of this term can be extended to any dynamic system. A standard and reasonable definition of a HDS would be to consider the structure only to indicate the objective to be reached and the fixed terminology. So, in this paper, one will restricts to a particular class of HDS, namely the switched systems, which constitute a set of continuous subsystems and a switching rule that orchestrates the commutation between them [5],[7],[10],[18].

The various studies dedicated to switched systems consider mainly the stability problem. Indeed, three basic problems involved in the stability of the switched system were raised by Liberzon [7] and then developed in the literature [13],[15]. One of these problems is to find the conditions that guarantee the system's asymptotic stability under any switching rule. i.e. arbitrary switching sequence. A necessary condition for asymptotic stability under arbitrary switching sequence is that each sub-system must be asymptotically stable. Authors showed in [15] that this condition is not sufficient, since, it is possible to lead to instability for some classes of switching signals. Thus, to solve this problem, it has been shown in [8] that the existence of a common Lyapunov function is a necessary and sufficient condition. This approach is also reported in several works, see [10-12],[16],[17],[1].

This paper deals primarily with the last problem described above. It concerns the class of continuous-time uncertain, switched linear systems without jump at the switching instant. It is also assumed that the number of switching is finite in a finite time (non zenon). In [9], quasi LMI condition ensuring the design of a control law for disturbed 
linear switched system is proposed. In this paper, the robustness of the approach has been improved by considering an uncertain system. In fact, in this case it is not obvious to get strict LMI condition for the asymptotic stability. Thus, the goal of this paper is to provide strict LMI condition for the synthesizes of a switched state feedback control law that guarantees asymptotic stability of the closed loop-system for any arbitrary switching rule and ensures three different performances at the same time. First, the asymptotic stability with some guaranteed specifications, namely; the transient response and the damping factor [6] by the poles location of each closed-loop linear subsystems. The second performance is to attenuate the disturbance according to $\mathrm{H} \infty$ criterion. And finally, the main contribution of this paper is to obtain state feedback gains thank to the LMI formulation by considering an uncertain structure of the switched system. A numerical example will illustrate the proposed approach.

\section{Uncertain switched systems}

Let us consider the following uncertain switched system:

$$
\left\{\begin{array}{l}
\dot{x}(t)=A_{\sigma(t)}(t) x(t)+B_{1 \sigma(t)}(t) \varphi(t)+B_{2 \sigma(t)}(t) u(t) \\
y(t)=C_{\sigma(t)}(t) x(t)+D_{1 \sigma(t)}(t) \varphi(t)+D_{2 \sigma(t)}(t) u(t)
\end{array}\right.
$$

with $A_{\sigma(t)}(t)=A_{\sigma(t)}+\Delta A_{\sigma(t)}(t), B_{1 \sigma(t)}(t)=B_{1 \sigma(t)}+\Delta B_{1 \sigma(t)}(t)$, $B_{2 \sigma(t)}(t)=B_{2 \sigma(t)}+\Delta B_{2 \sigma(t)}(t), C_{\sigma(t)}(t)=C_{\sigma(t)}+\Delta C_{\sigma(t)}(t), D_{1 \sigma(t)}(t)=D_{1 \sigma(t)}+\Delta D_{1 \sigma(t)}(t)$, $D_{2 \sigma(t)}(t)=D_{2 \sigma(t)}+\Delta D_{2 \sigma(t)}(t)$ and where $\sigma(t)$ is the switching rule defined as follows:

Let $\mathrm{I}=\{1,2, \ldots, N\}$ an index's compact set of the sub-systems, $\sigma(t)$ is defined by the mapping: $\sigma(t): \square^{+} \rightarrow I$, i.e. the linear mode $\left(A, B_{1}, B_{2}, C, D_{1}, D_{2}\right)_{l}$, is active if $\sigma(t)=l$ with $l \in I$. 
$x(t) \in \square^{n}$ is the state vector, $u(t) \in \square^{m}$ is the control input vector, $\varphi(t) \in \square^{r}$ is an exogenous input, $A_{\sigma(t)} \in \square^{n \times n}, B_{1 \sigma(t)} \in \square^{n \times r}, B_{2 \sigma(t)} \in \square^{n \times m}, D_{1 \sigma(t)} \in \square^{p \times r}, D_{2 \sigma(t)} \in \square^{p \times m}$ and $C_{\sigma(t)} \in \square^{p \times n}$ are the subsystem matrices. $\Delta A_{\sigma(t)}(t), \Delta B_{1 \sigma(t)}(t), \Delta B_{2 \sigma(t)}(t)$, $\Delta D_{1 \sigma(t)}(t), \Delta D_{2 \sigma(t)}(t)$ and $\Delta C_{\sigma(t)}(t)$ contain all the modelling uncertainties, which can be represented by:

$$
\begin{aligned}
& \Delta A_{\sigma(t)}(t)=H_{a \sigma(t)} \Delta_{a \sigma(t)}(t) N_{a \sigma(t)}, \Delta B_{1 \sigma(t)}(t)=H_{1 b \sigma(t)} \Delta_{1 b \sigma(t)}(t) N_{1 b \sigma(t)}, \\
& \Delta B_{2 \sigma(t)}(t)=H_{2 b \sigma(t)} \Delta_{2 b \sigma(t) \sigma(t)}(t) N_{2 b \sigma(t)}, \Delta D_{1 \sigma(t)}(t)=H_{1 d \sigma(t)} \Delta_{1 d \sigma(t)}(t) N_{1 d \sigma(t)}, \\
& \Delta D_{2 \sigma(t)}(t)=H_{2 d \sigma(t)} \Delta_{2 d \sigma(t)}(t) N_{2 d \sigma(t)} \text { and } \Delta C_{\sigma(t)}(t)=H_{c \sigma(t)} \Delta_{c \sigma(t)}(t) N_{c \sigma(t)} .
\end{aligned}
$$

where $H_{a \sigma}, H_{1 b \sigma}, H_{2 b \sigma}, H_{1 d \sigma}, H_{2 d \sigma}, H_{c \sigma}, N_{a \sigma}, N_{1 b \sigma}, N_{2 b \sigma}, N_{1 d \sigma}, N_{2 d \sigma}$ and $N_{c \sigma}$ are constant matrices and, $\Delta_{a \sigma(t)}(t), \Delta_{1 b \sigma(t)}(t), \Delta_{2 b \sigma(t)}(t), \Delta_{1 d \sigma(t)}(t), \quad \Delta_{2 d \sigma(t)}(t)$ and $\Delta_{c \sigma(t)}(t)$ are the normalized uncertainty matrices, verifying the following conditions:

$$
\begin{aligned}
& \Delta_{a \sigma(t)}^{T}(t) \Delta_{a \sigma(t)}(t) \leq I, \Delta_{1 b \sigma(t)}^{T}(t) \Delta_{1 b \sigma(t)}(t) \leq I, \underset{2 b \sigma(t)}{T}(t) \Delta_{2 b \sigma(t)}(t) \leq I, \\
& \Delta_{1 d i}^{T}(t) \Delta_{1 d i}(t) \leq I, \Delta_{2 d \sigma(t)}^{T}(t) \Delta_{2 d \sigma(t)}(t) \leq I \text { and } \Delta_{c \sigma(t)}^{T}(t) \Delta_{c \sigma(t)}^{T}(t) \leq I .
\end{aligned}
$$

In this study, we assume that the state vector is available to perform a state-feedback and that the discrete control law $\sigma(t)$ is not a priori known but is available in real time. Now, we consider the following state feedback control law:

$$
u(t)=K_{\sigma(t)} x(t)
$$

From (1) and (2) the closed loop switched system can be written as follow:

$$
\left\{\begin{array}{l}
\dot{x}(t)=\tilde{A}_{\sigma}(t) x(t)+B_{1 \sigma}(t) \varphi(t) \\
y(t)=\tilde{C}_{\sigma}(t) x(t)+D_{1 \sigma}(t) \varphi(t)
\end{array}\right.
$$


where :

$$
\begin{aligned}
& \tilde{A}_{\sigma}(t)=\bar{A}_{\sigma}(t)+\bar{B}_{2 \sigma}(t) K_{\sigma} \\
& \tilde{C}_{\sigma}(t)=\bar{C}_{\sigma}(t)+\bar{D}_{2 \sigma}(t) K_{\sigma}
\end{aligned}
$$

The objective is then to determine the state feedback gains $K_{\sigma}$ ensuring the stability of the closed-loop switched system (3)

\section{Robust pole placement and $\mathrm{H} \infty$ performances}

In a first step, one determines the state feedback gains with poles placement of each closed loop uncertain linear subsystem, such that the eigenvalues of $\tilde{A}_{\sigma}(t)$ are located inside a disk-pole with centre at $\left(-\left(r_{\sigma}+d_{\sigma}\right), 0\right)$ with the radius $r_{\sigma}$ and the distance $d_{\sigma}$ from the imaginary axis as set in figure 1.

Using the parameter $d_{\sigma}$, it is possible to determine an upper bound for the settling time of the transient response given by $3 \tau_{\sigma}$ or $5 \tau_{\sigma}$ for each sub-system $\sigma$, with $\tau_{\sigma}=1 / d_{\sigma}$. The value $r_{\sigma}$ gives the upper bound on the natural frequency of oscillation for the transient response. Notice also that a lower bound on the dumping factor $\xi_{\sigma}$, which determines the overshoot, can be computed as follows [9]:

$\xi_{\sigma}=\frac{\sqrt{\left(r_{\sigma}+d_{\sigma}\right)^{2}-r_{\sigma}^{2}}}{r_{\sigma}+d_{\sigma}}$

Using the well known common quadratic Lyapunov function:

$V(x)=x^{T} P x, P=P^{T}>0$ 
a necessary and sufficient condition such that all the eigenvalues of $\tilde{A}_{\sigma}(t)$ of the system (3) lie inside the circular region $\varsigma\left(d_{\sigma}, r_{\sigma}\right)$ as depicted in figure 1 , is given by the existence of a positive definite symmetric matrix $W=P^{-1}$ such that the following inequality holds [3]:

$$
\begin{aligned}
& \left(\tilde{A}_{\sigma}{ }^{T}(t)+d_{\sigma} I\right) W+W\left(\tilde{A}_{\sigma}(t)+d_{\sigma} I\right)^{T}+\frac{1}{r_{\sigma}}\left(\tilde{A}_{\sigma}(t)+d_{\sigma} I\right) W\left(\tilde{A}_{\sigma}(t)+d_{\sigma} I\right)^{T}<0 \\
& \sigma=1,2, \ldots, N
\end{aligned}
$$

In the other hand, in a second step, one needs to attenuate the disturbances $\varphi(t)$ with a minimum rate $\eta$, according to the $\mathrm{H} \infty$ criterion:

$$
\|y(t)\|_{2} \leq \eta\|\varphi(t)\|_{2}
$$

A sufficient condition ensuring the $\mathrm{H} \infty$ criterion (8) is given by the following matrix inequality [4]:

$$
\left[\begin{array}{ccc}
\tilde{A}_{\sigma}(t) W+W \tilde{A}_{\sigma}{ }^{T}(t) & (*) & (*) \\
B_{1 \sigma}{ }^{T}(t) & -I & (*) \\
\tilde{C}_{\sigma}(t) W & D_{1 \sigma}(t) & -\eta^{2} I
\end{array}\right]<0, \sigma=1,2, \ldots, N
$$

Finally, to achieve both the performances at the same time; i.e. disturbance's attenuation according to the $\mathrm{H} \infty$ criterion and pole placements (inside the disk-pole $\varsigma\left(d_{\sigma}, r_{\sigma}\right)$ ) leading to a condition that verifies both (7) and (9), we use the following lemma [9]:

Lemma 1: If there exists a symmetric positive definite matrix $W$ such that:

$$
\left[\begin{array}{cccc}
\tilde{A}_{\sigma}(t) W+W \tilde{A}_{\sigma}{ }^{T}(t)+2 d_{\sigma} W & (*) & (*) & (*) \\
W \tilde{A}_{\sigma}{ }^{T}(t)+d_{\sigma} W & -r_{\sigma} W & 0 & 0 \\
B_{1 \sigma}{ }^{T}(t) & 0 & -I & (*) \\
\tilde{C}_{\sigma}(t) W & 0 & D_{1 \sigma}(t) & -\eta^{2} I
\end{array}\right]<0, \sigma=1,2, \ldots, N
$$


Then, the stability of the closed-loop switched system (3) is ensured under arbitrary switching rule with guaranteed disturbance attenuation level $\eta$, defined in (8), and the pole location of each linear subsystem inside the disk-pole $\varsigma\left(d_{\sigma}, r_{\sigma}\right)$ depicted in figure 1 .

\section{Proof:}

Let us consider the following inequality:

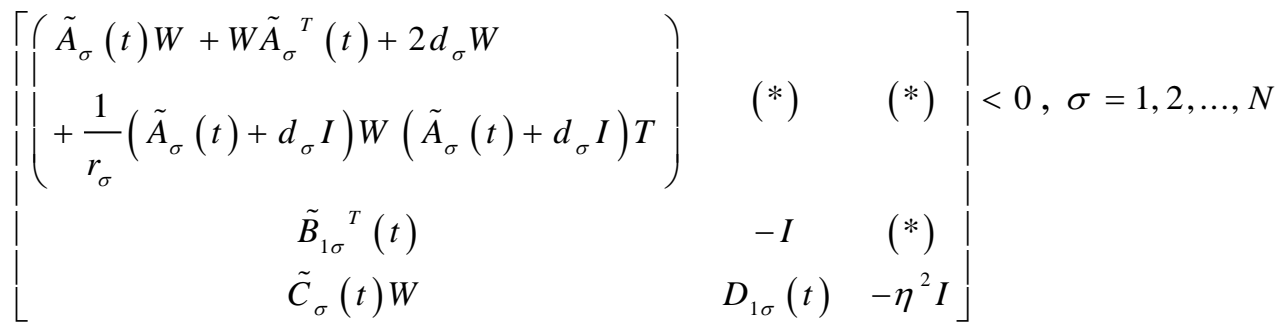

A necessary condition such that the inequality (11) holds is that the first diagonal block of (11) must be negative definite, i.e.:

$$
\tilde{A}_{\sigma}(t) W+W \tilde{A}_{\sigma}^{T}(t)+2 d_{\sigma} W+\frac{1}{r_{\sigma}}\left(\tilde{A}_{\sigma}(t)+d_{\sigma} I\right) W\left(\tilde{A}_{\sigma}(t)+d_{\sigma} I\right)^{T}<0
$$

That is equivalent to:

$$
\tilde{A}_{\sigma}(t) W+W \tilde{A}_{\sigma}^{T}(t)<-2 d_{\sigma} W-\frac{1}{r_{\sigma}}\left(\tilde{A}_{\sigma}(t)+d_{\sigma} I\right) W\left(\tilde{A}_{\sigma}(t)+d_{\sigma} I\right)^{T}<0
$$

leading to the inequality (9) of the Hoo. Then, by applying the Shur complement in the block $(1,1)$ of the inequality $(11)$, the inequality (10) is obtained. This end the proof.

Note that, the inequality (11) given by lemma 1 is not a LMI condition since, in our case, it contains varying time uncertainties which can be considered unknown with 
known upper bounds. Thus, in the following section, the goal is to get a LMI constraint by considering the uncertainties structure mentioned in the first section.

\section{LMI FORMULATION}

Because of the uncertainties, cheeking a LMI condition for the switched system, with performances stipulated above, is not trivial. In order to obtain a LMI condition to synthesize the state feedback gains, one needs to use the following corollaries:

Corollary 1[19]: For real matrices $A, B$ and $S=S^{T}>0$ with appropriate dimensions and a positive constant $\tau$, we have:

$A^{T} B+B^{T} A \leq \gamma A^{T} A+\gamma^{-1} B^{T} B$

and $X^{T} Y+Y^{T} X \leq X^{T} S^{-1} X+Y^{T} S Y$

Corollary 2: for real matrices $A, B, W, Y, Z$ and a regular matrix $Q$ with appropriate dimensions we have:

$\left[\begin{array}{cc}Y & W^{T}+B^{T} A^{T} \\ W+A B & Z\end{array}\right]<0 \Leftrightarrow\left[\begin{array}{cc}Y+B^{T} Q^{-1} B & W^{T} \\ W & Z+A Q A^{T}\end{array}\right]<0$

Proof of corollary 2 : For real matrices $A, B, W, Y, Z$ and a regular matrix $Q$ with appropriate dimensions, the matrix:

$\left[\begin{array}{cc}Y & W^{T}+B^{T} A^{T} \\ W+A B & Z\end{array}\right]<0$ can be rewritten as: $\left[\begin{array}{cc}Y & W^{T} \\ W & Z\end{array}\right]+\left[\begin{array}{cc}0 & B^{T} A^{T} \\ A B & 0\end{array}\right]<0$

From the inequality (13) we have: 
$\left[\begin{array}{l}0 \\ A\end{array}\right]\left[\begin{array}{ll}B & 0\end{array}\right]+\left[\begin{array}{c}B^{T} \\ 0\end{array}\right]\left[\begin{array}{ll}0 & A^{T}\end{array}\right] \leq\left[\begin{array}{l}0 \\ A\end{array}\right] M\left[\begin{array}{ll}0 & A^{T}\end{array}\right]+\left[\begin{array}{c}B^{T} \\ 0\end{array}\right] M^{-1}\left[\begin{array}{ll}B & 0\end{array}\right]$

that leads to (15) and end the proof.

Now, let us make a bijective change of variable on the state feedback gains; $Z_{\sigma}=K_{\sigma} W$. Then, the main result can be summarized by the following theorem:

Theorem 1: If there exists a symmetric positive definite matrix $W$, matrices $Z_{\sigma}$ and positive constants $\tau_{1} \tau_{2}, \tau_{3} \tau_{4}, \tau_{5} \tau_{6}, \tau_{7}$ such that:

$\left[\begin{array}{c:c}\Xi & (*) \\ \hdashline \Sigma & \Psi\end{array}\right]<0$

with:

$\Xi=A_{\sigma} W+B_{2 \sigma} Z_{\sigma}+W A_{\sigma}{ }^{T}+Z_{\sigma}{ }^{T} B_{2 \sigma}{ }^{T}+2 d_{\sigma} W+\left(\tau_{1}{ }^{-1}+\tau_{3}{ }^{-3}\right) H_{a \sigma} H_{a \sigma}{ }^{T}+\left(\tau_{2}{ }^{-1}+\tau_{4}{ }^{-1}\right) H_{2 b \sigma} H_{2 b \sigma}{ }^{T}$ $\left.\Sigma=\mid \begin{array}{c}N_{1 b \sigma} \\ N_{2 b \sigma} Z_{\sigma} \\ N_{c \sigma} W \\ N_{2 d \sigma} Z_{\sigma} \\ N_{a \sigma} W \\ W A_{\sigma}{ }^{T}+Z_{\sigma}{ }^{T} B_{2 \sigma}{ }^{T}+d_{\sigma} W \\ 0 \\ 0 \\ B_{1 \sigma}{ }^{T} \\ C_{\sigma}+D_{2 \sigma} Z_{\sigma} \\ 0\end{array}\right]$ 
and $\Psi=\left[\begin{array}{c:cccccc}\Psi_{1,1} & \multicolumn{7}{c}{0} & 0 & 0 \\ \hdashline & N_{a \sigma} W & -\tau_{3}{ }^{-1} & 0 & 0 & 0 & 0 \\ & N_{2 b \sigma} Z_{\sigma} & 0 & -\tau_{4}^{-1} & 0 & 0 & 0 \\ 0 & 0 & 0 & 0 & \Psi_{5,5} & (*) & 0 \\ & 0 & 0 & 0 & D_{1 \sigma} & \Psi_{6,6} & (*) \\ & 0 & 0 & 0 & 0 & H_{1 d \sigma}^{T} & -\tau_{8}^{-1}\end{array}\right]$,

and where $\Psi_{1,1}=\operatorname{diag}\left[\begin{array}{lllll}-\tau_{5} & -\tau_{2}^{-1} & -\tau_{6} & -\tau_{7} & -\tau_{1}^{-1}\end{array}\right]$,

$\Psi_{5,5}=-I+\tau_{5} H_{1 b \sigma} H_{1 b \sigma}^{T}+\tau_{8}^{-1} N_{1 d \sigma}^{T} N_{1 d \sigma}, \Psi_{6,6}=-\eta^{2} I+\tau_{6} H_{c \sigma} H_{c \sigma}^{T}+\tau_{7} H_{2 d \sigma} H_{2 d \sigma}^{T}$

for $\sigma=1,2, \ldots, N$.

Then, the quadratic stability of the closed-loop switched system (3) is ensured under an arbitrary switching rule, with guaranteed disturbance attenuation level $\eta$ and with the pole location of each linear subsystem inside the disk-pole $\varsigma\left(d_{\sigma}, r_{\sigma}\right)$ depicted in figure 1 .

\section{Proof:}

Starting from the inequality (10) and separating the uncertainties, (10) becomes:

$\Pi+\Delta \Pi<0$

with :

$\Pi=\left[\begin{array}{cccc}\left(A_{\sigma}+B_{2 \sigma} K_{\sigma}\right) W+(*)+2 d_{\sigma} W & (*) & (*) & (*) \\ W\left(A_{\sigma}+B_{2 \sigma} K_{\sigma}\right)^{T}+d_{\sigma} W & -r_{\sigma} W & 0 & 0 \\ B_{1 \sigma}{ }^{T} & 0 & -I & (*) \\ \left(C_{\sigma}+D_{2 \sigma} K_{\sigma}\right) W & 0 & D_{1 \sigma} & -\eta^{2} I\end{array}\right]$ 
and $\Delta \Pi=\left[\begin{array}{cccc}\left(\Delta A_{\sigma}(t)+\Delta B_{2 \sigma}(t) K_{\sigma}\right) W+(*) & (*) & (*) & (*) \\ W\left(\Delta A_{\sigma}(t)+\Delta B_{2 \sigma}(t) K_{\sigma}\right)^{T} & 0 & 0 & 0 \\ \Delta B_{1 \sigma}{ }^{T}(t) & 0 & 0 & (*) \\ \left(\Delta C_{\sigma}(t)+\Delta D_{2 \sigma}(t) K_{\sigma}\right) W & 0 & \Delta D_{1 \sigma}(t) & 0\end{array}\right]$.

After the bijective change of variable $Z_{\sigma}=K_{\sigma} W$, one obtains:

$\Pi=\left[\begin{array}{cccc}A_{\sigma} W+(*)+B_{2 \sigma} Z_{\sigma}+(*)+2 d_{\sigma} W & (*) & (*) & (*) \\ W A_{\sigma}{ }^{T}+Z_{\sigma}{ }^{T} B_{2 \sigma}{ }^{T}+d_{\sigma} W & -r_{\sigma} W & 0 & 0 \\ B_{1 \sigma}{ }^{T} & 0 & -I & (*) \\ C_{\sigma} W+D_{2 \sigma} Z_{\sigma} & 0 & D_{1 \sigma} & -\eta^{2} I\end{array}\right]$

and $\Delta \Pi=\left[\begin{array}{cccc}\Delta A_{\sigma}(t) W+(*)+\Delta B_{2 \sigma}(t) Z_{\sigma}+(*) & (*) & (*) & (*) \\ W \Delta A_{\sigma}{ }^{T}(t)+Z_{\sigma}{ }^{T} \Delta B_{2 \sigma}{ }^{T}(t) & 0 & 0 & 0 \\ \Delta B_{1 \sigma}{ }^{T}(t) & 0 & 0 & (*) \\ \Delta C_{\sigma}(t) W+\Delta D_{2 \sigma}(t) Z_{\sigma} & 0 & \Delta D_{1 \sigma}(t) & 0\end{array}\right]$.

Holding the uncertainties structure shown in section I, thus $\Delta \Pi$ is written as:

$\Delta \Pi=\left[\begin{array}{cccc}\Delta \Pi_{1,1} & (*) & (*) & (*) \\ \Delta \Pi_{2,1} & 0 & 0 & 0 \\ \Delta \Pi_{3,1} & 0 & 0 & (*) \\ \Delta \Pi_{4,1} & 0 & H_{1 d} \Delta_{1 d \sigma}(t) N_{1 d \sigma} & 0\end{array}\right]$,

$\Delta \Pi_{1,1}=H_{a \sigma} \Delta_{a \sigma}(t) N_{a \sigma} W+(*)+H_{2 b} \Delta_{2 b \sigma}(t) N_{2 b} Z_{\sigma}+(*)$,

$\Delta \Pi_{2,1}=W N_{a \sigma}^{T} \Delta_{a \sigma}^{T}(t) H_{a \sigma}^{T}+Z_{\sigma}^{T} N_{2 b \sigma}^{T} \Delta_{2 b \sigma}^{T}(t) H_{2 b \sigma}^{T}, \Delta \Pi_{3,1}=H_{1 b \sigma} \Delta_{1 b \sigma}(t) N_{1 b \sigma}$

and $\Delta \Pi_{4,1}=H_{c \sigma} \Delta_{c \sigma}(t) N_{c \sigma} W+H_{2 d \sigma} \Delta_{2 d \sigma}(t) N_{2 d \sigma} Z_{\sigma}$.

Now, by the means of corollaries 1 and 2 , the matrix $\Delta \Pi$ containing the uncertainties can be bounded in order to find some scalar constants as following:

$\Delta \Pi<\operatorname{diag}[\Gamma]$ 
where:

$$
\begin{aligned}
\Gamma_{1,1} & =\tau_{1} W N_{a \sigma}^{T} N_{a \sigma} W+\tau_{1}^{-1} H_{a \sigma} H_{a \sigma}^{T}+\tau_{2} Z_{\sigma}{ }^{T} N_{2 b \sigma}^{T} N_{2 b \sigma} Z_{\sigma} \\
& +\tau_{2}{ }^{-1} H_{2 b \sigma} H_{2 b \sigma}^{T}+\tau_{3}{ }^{-3} H_{a \sigma} H_{a \sigma}^{T}+\tau_{4}{ }^{-1} H_{2 b \sigma} H_{2 b \sigma}^{T} \\
& +\tau_{5}{ }^{-1} N_{1 b \sigma}^{T} N_{1 b \sigma}+\tau_{6}{ }^{-1} W N_{c \sigma}^{T} N_{c \sigma} W+\tau_{7}{ }^{-1} Z_{\sigma}{ }^{T} N_{2 d \sigma}^{T} N_{2 d \sigma} Z_{\sigma} \\
\Gamma_{2,2} & =\tau_{3} W N_{a \sigma}^{T} N_{a \sigma} W+\tau_{4} Z_{\sigma}^{T} N_{2 b}^{T} N_{2 b} Z_{\sigma}, \Gamma_{3,3}=\tau_{5} H_{1 b \sigma} H_{1 b \sigma}^{T}+\tau_{8}{ }^{-1} N_{1 d \sigma}^{T} N_{1 d \sigma}
\end{aligned}
$$

and $\Gamma_{4,4}=\tau_{6} H_{c \sigma} H_{c \sigma}^{T}+\tau_{7} H_{2 d \sigma} H_{2 d \sigma}^{T}+\tau_{8} H_{1 d \sigma} H_{1 d \sigma}^{T}$.

Finally, by adding matrices $\Pi$ and $\Delta \Pi$, and using the Shur complement on the diagonal block terms, one finds easily LMI conditions of theorem 1.

\section{EXAMPLE AND SIMULATION}

To illustrate the results of theorem 2, we consider a numerical example that is composed of the following matrices:

$$
\begin{aligned}
& A_{1}=\left[\begin{array}{cc}
0 & 1 \\
-3 & -0.2
\end{array}\right], A_{2}=\left[\begin{array}{cc}
-1 & 1 \\
2 & 0
\end{array}\right], \\
& \Delta A_{1}(t)=\left[\begin{array}{cc}
0 & 0 \\
0.0005 \sin (t) & 0
\end{array}\right]=\left[\begin{array}{c}
0 \\
0.05
\end{array}\right] \sin (t)\left[\begin{array}{ll}
0.01 & 0
\end{array}\right]=H_{a 1} \Delta_{a 1}(t) N_{a 1}, \\
& \Delta A_{2}(t)=\left[\begin{array}{cc}
0.001 \cos (t) & 0 \\
0 & 0
\end{array}\right]=\left[\begin{array}{c}
0.1 \\
0
\end{array}\right] \cos (t)\left[\begin{array}{ll}
0.01 & 0
\end{array}\right]=H_{a 2} \Delta_{a 2}(t) N_{a 2}, \\
& B_{11}=B_{12}=I_{2 \times 2}, \Delta B_{11}(t)=\Delta B_{12}(t)=0_{2 \times 2}, B_{21}=\left[\begin{array}{l}
0 \\
1
\end{array}\right], B_{22}=\left\lceil\begin{array}{l}
1 \\
0
\end{array}\right], \\
& \Delta B_{21}(t)=\left[\begin{array}{c}
0 \\
0.01 \cos (t)
\end{array}\right]=\left[\begin{array}{c}
0 \\
0.01
\end{array}\right] \cos (t)=H_{2 b 1} \Delta_{2 b 1}(t) N_{2 b 1}, \\
& \Delta B_{22}(t)=\left[\begin{array}{c}
0.01 \sin (t) \\
0
\end{array}\right]=\left[\begin{array}{c}
0.01 \\
0
\end{array}\right] \sin (t)=H_{2 b 2} \Delta_{2 b 2}(t) N_{2 b 2},
\end{aligned}
$$


$C_{1}=C_{2}=\left[\begin{array}{ll}1 & 0\end{array}\right], \Delta C_{1}(t)=\Delta C_{2}(t)=0_{1 \times 2}, D_{11}=D_{12}=0_{2 \times 1}, \Delta D_{11}(t)=\Delta D_{12}(t)=0_{2 \times 1}$, $D_{21}=D_{22}=1$ and $\Delta D_{21}(t)=\Delta D_{22}(t)=0$.

The external disturbance of the switched system is given by the vector: $\varphi(t)=0.001[\sin (2 t) 2 \cos (2 t)]$, this one is attenuated at the level $\eta=0.45$. By choosing the following specifications: $r_{1}=1, r_{2}=1.5, d_{1}=0.9, \quad d_{2}=1.2$, corresponding to the radius and the distance of the disk-pole from the origin of complex plan for the pole of each sub-systems as depicted in figure 1.

After solving the LMI (17) of theorem 1, one obtains the following state feedback gains: $K_{1}=\left[\begin{array}{ll}0.8516 & -2.6653\end{array}\right], \quad K_{2}=\left[\begin{array}{ll}-3.0497 & -2.8451\end{array}\right]$ and the matrix $P=W^{-1}=\left[\begin{array}{ll}0.1812 & 0.1240 \\ 0.1240 & 0.0958\end{array}\right]$.

The switched system is subjected to a known commutation law, whose rule consists in fixing a dwell time for each one. We choose to switch regularly at every 0.25 seconds from one mode of operation to another by starting from sub-system 2, as illustrated in figure 5 .

The initial state conditions are fixed at $x_{1}(0)=1, x_{2}(0)=0$ and represented by $*$ in the phase diagram, figure 3 . Thus, this shows the convergence of the system to the equilibrium point.

The system is stabilised with a settling time less than four seconds as depicted in figure 2. Finally, the control signal is illustrated by figure 4 . Figure 5 shows the switching signal.

\section{CONCLUSION}

This work deals with robust control synthesis for a class of hybrid dynamical system. This one concerns a set of linear uncertain and switched systems with arbitrary 
switching rules. In fact, based on a state feedback control, we have developed sufficient conditions in term of LMI that ensures the attenuation of the external disturbances according to $\mathrm{H} \infty$ criterion. Then, robust poles placements according to the desired specifications, namely, the damping factor and time response of each sub-system, were considered. Our future work intends to consider that the states are not available for measurements to get LMI conditions in the case of hybrid observer synthesis.

\section{References}

[1] D. Angeli, «A note on stability of arbitrarily switched homogeneous systems », Systems and Control Letters, Nonlinear Control Abstracts 2000.

[2] P. J. Antsaklis, « Hybrid Systems: Theory \& Applications », Proceedings of the IEEE - Special Issue on, volume 88 of 12. July 2000.

[3] J. Bernussou and G. Garcia. «Disk pole assignment for uncertain systems with norm bounded uncertainty », IFAC International Workshop on Robust Control, airns Queensland Australia 1993.

[4] S. Boyd, L. El Ghaoui, E. Feron, V. Balakrishnan, « Linear Matrix Inequalities in System and Control Theory », SIAM Studies in Applied Mathematics, Philadelphia, PA, 1994.

[5] M.S. Branicky, «Multiple lyapunov functions and other analysis tools for switched and hybrid systems », IEEE Transactions on Automatic Control, 43(4):475482, April 1998

[6] W.M. Haddad, D.S. Bernstein, «Controller design with regional pole constraints », IEEE Trans. Automat. Control 37 (1) (1992) 54-69.

[7] D. Liberzon and A.S. Morse, «Basic problems in stability and design of switched systems », IEEE Control Systems, 19(5):59-70, October 1999.

[8] Y. Lin, E. D. Sontag and Y.Wang, «A Smooth converse Lyapunov theorem for robust stability », SIAM Journal on Control and Optimization, 34, 1996, pp. 124-160.

[9] V.F. Montagner, V. J. S. Leite, R. C. L. F. Oliveira, P. L. D, «Peres. State feedback control of switched linear systems: An LMI approach », Journal of Computational and Applied Mathematics 194 (2006) 192-206 
[10] Y. Mori, T. Mori, and Y. Kuroe, « On a class of linear constant systems which have a common quadratic Lyapunov function », 37th IEEE Conference on Decision and Control, pages 2808-2809, 1998.

[11] K.S. Narendra and J. Balakrishnan, « A common lyapunov function for stable lti systems with commuting a-matrices », IEEE Transactions on Automatic Control, 39:2469-2471, 1994

[12] T. Ooba and Y. Funahashi, «Two conditions concerning common quadratic lyapunov functions for linear systems », IEEE Transactions on Automatic Control, 42:719-721, 1997.

[13] P. Peleties and R. DeCarlo, «Asymptotic stability of m-switched systems using Lyapunov-like functions », American Control Conference, pages 1679-1684, 1991

[14] S. Pettersson, «Analysis and design of hybrid systems », $\mathrm{PhD}$ thesis, Chalmers University of Technology, Sweden, 1999

[15] A. V. Schaft and H. Shumacher, «An Introduction to Hybrid Dynamical Systems », Springer, Lecture Notes in Control and information Sciences.volume 251, , 2000

[16] H. Shim, D. J. Noh, and J. H.Seo, «Common Lyapunov function for exponentially stable nonlinear systems », 14th SIAM Conf. On Control and its Applications, Jaksonville, FL, 1998.

[17] R. N. Shorten and K. S, Narenda, «A sufficient condition for the existence of a common Lyapunov function for two second-order linear »,

[18] X. Xu, «Analysis and design of switched systems », PhD thesisUniversity of Notre Dame, Indiana 2001

[19] K. Zhou, P. P. Khargonedkar, «Robust stabilization of linear systems with norm-bounded time-varying uncertainty », Syst. Control Letters, Vol. 10, 1988, pp. 1720. 


\section{LIST OF FIGURES}

Figure1: Pole placement for the sub-system $\sigma$

Figure 2. State trajectories $\mathrm{x}_{1}$ and $\mathrm{x}_{2}$

Figure 3. Phase plan

Figure 4. Control input signal

Figure 5. Switching signal 


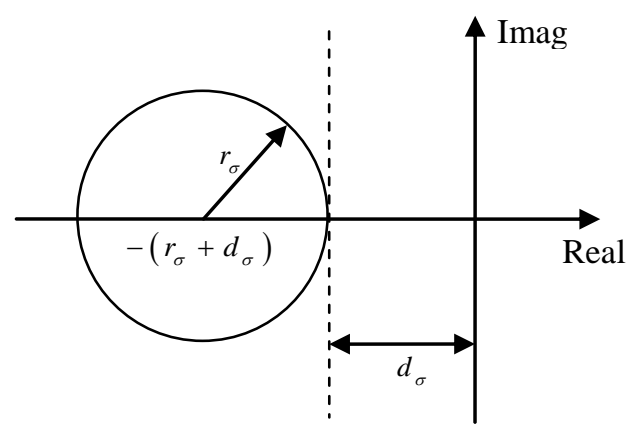

Fig 1: Pole placement for the sub-system $\sigma$

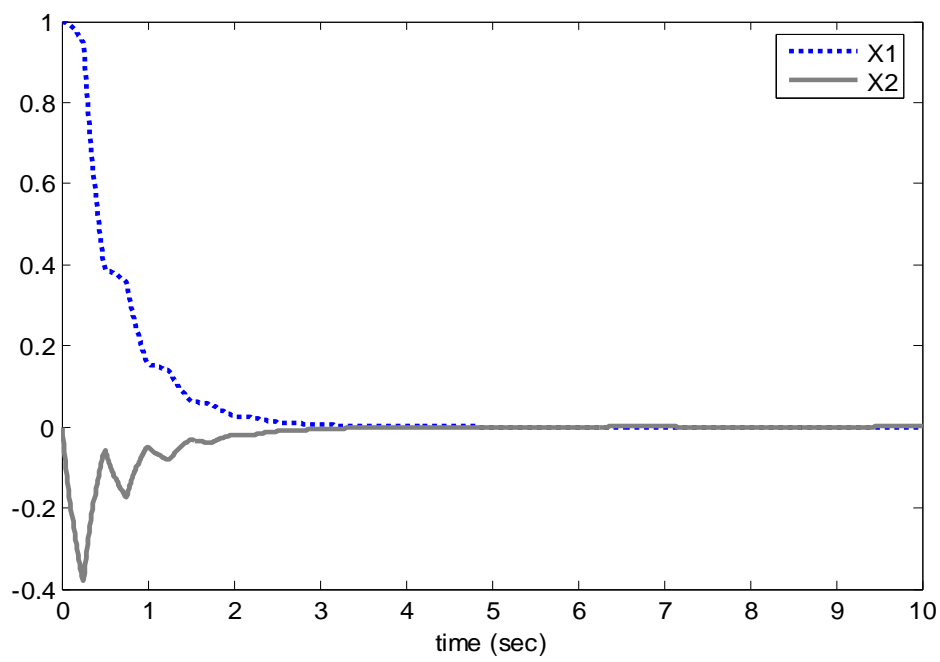

Figure 2. State trajectories $\mathrm{x}_{1}$ and $\mathrm{x}_{2}$ 


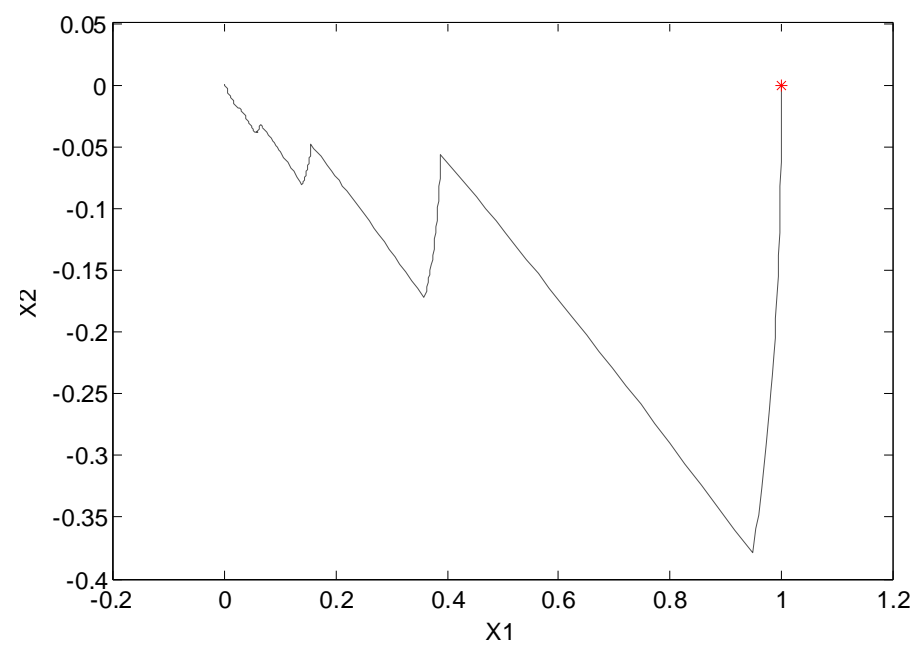

Figure 3. Phase plan

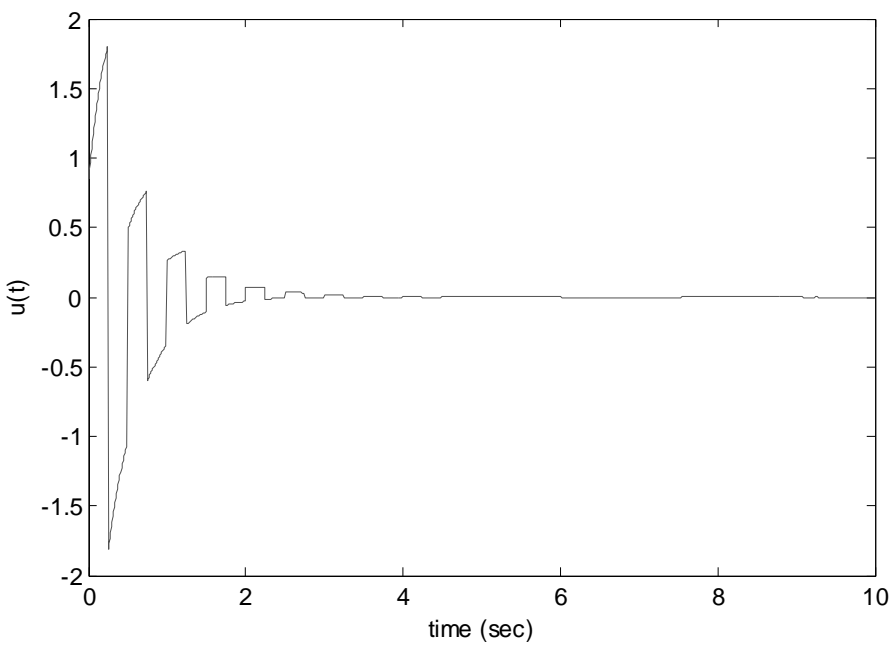

Figure 4. Control input signal 


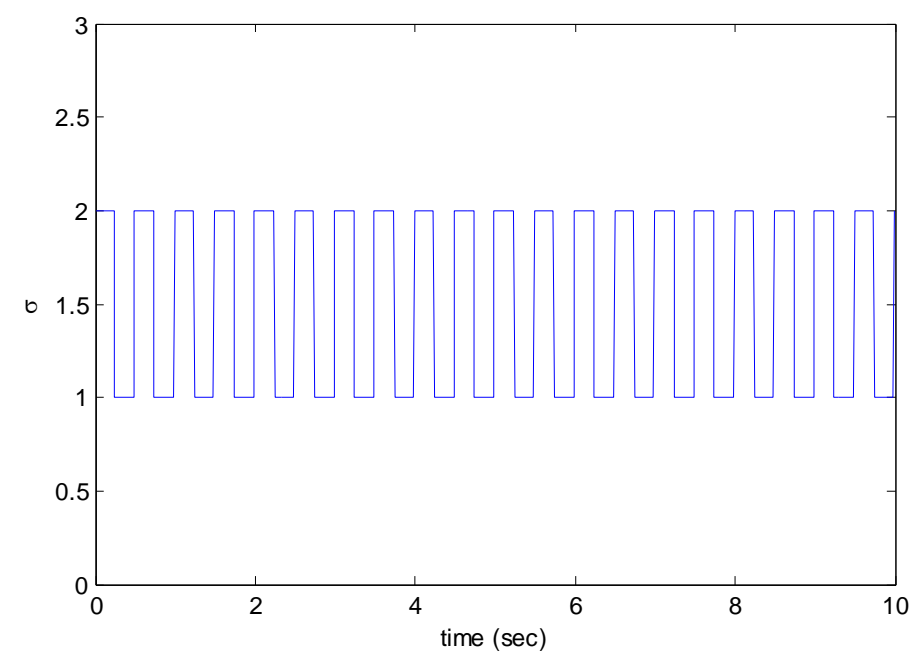

Figure 5. Switching signal 